\title{
FAST AND ROBUST STABILITY REGION ESTIMATION FOR NONLINEAR DYNAMICAL SYSTEMS
}

\author{
ELOÏSE BERTHIER, JUSTIN CARPENTIER AND FRANCIS BACH \\ Inria - Ecole Normale Supérieure \\ PSL Research University, Paris, France
}

\begin{abstract}
A linear quadratic regulator can stabilize a nonlinear dynamical system with a local feedback controller around a linearization point, while minimizing a given performance criteria. An important practical problem is to estimate the region of attraction of such a controller, that is, the region around this point where the controller is certified to be valid. This is especially important in the context of highly nonlinear dynamical systems. In this paper, we propose two stability certificates that are fast to compute and robust when the first, or second derivatives of the system dynamics are bounded. Associated with an efficient oracle to compute these bounds, this provides a simple stability region estimation algorithm compared to classic approaches of the state of the art. We experimentally validate its application to both polynomial and non-polynomial systems of various dimensions, including standard robotic systems, for estimating region of attractions around equilibrium points, as well as for trajectory tracking.
\end{abstract}

\section{INTRODUCTION}

Controlling a robot typically involves a global motion planning to steer the system from an initial position to a target goal, as well as some local feedback corrections to accurately track the planned trajectory. For instance, the combination of rapidly-exploring random trees [LK01] and local trajectory stabilization led to a fruitful feedback motion planning algorithm named LQR-trees [TMTR10]. In this algorithm, a locally optimal trajectory is computed between sampled points of the state space. Each trajectory is then locally stabilized with a linear quadratic regulator (LQR). The aim is to design a global controller by covering the whole state space with overlapping funnels, i.e., regions of attraction (ROA) around trajectories. An important subproblem is to estimate such an ROA: a set of initial states that the controlled dynamics brings back to an equilibrium. Crucially, it must be performed efficiently, as it will be called repeatedly to cover a potentially large dimensional state space. Ideally, the estimation must be fast to compute, but not overly conservative.

A controlled dynamical system can be stabilized around an equilibrium point with an adequate closedloop controller. It is possible to synthesize an optimal feedback controller for some stability criterion [GSM90], but a simply available candidate is nothing more than the LQR. The stability of a region is commonly assessed with a Lyapunov function, which again can be optimized [GH15, Joh00], or not. This paper focuses on finding the largest estimate of the ROA for a given controller and a given Lyapunov function, both obtained from LQR. The gold standard technique for this problem is based on sum of squares (SOS) programming and provides high quality estimates. Yet it is limited to polynomial dynamics, and grows computationally heavy in large dimensions, hence limiting its applicability in practice, especially in the context of robotics where fast methods are needed to accurately control and stabilize the motions of the robot such as for legged locomotion [CM18b].

Another stake in robotics is robustness with respect to model misspecification or uncertainties. In particular, there can be a shift between the behavior of a simulated robotic system and its physical counterpart [SCH$\left.{ }^{+} 18\right]$. Robust ROA estimation methods [Che04] must account for the uncertainty on the parameters of the dynamics. In particular, we focus on the case where the Jacobian or the Hessian of the dynamics is known to be bounded. This applies to robust control, but also to perfectly known dynamics that are computationally hard to handle. Bounding the Jacobian or Hessian is possible analytically for some simple low-order polynomial systems, or by sampling, taking advantage of automatic differentiation for complicated robotic systems [GNS ${ }^{+}$17]. Interestingly, the bounds can be computed offline, in parallel, or

E-mail address: firstname. lastname@inria.fr. 
experimentally with a real physical system. With such information on the dynamics, our goal is to design fast, robust ROA estimation methods, practical in large state dimensions.

Our main contribution is to propose a general ROA estimation framework for non-polynomial systems, which is faster and simpler than SOS-based methods. The paper is organized as follows. After introducing the principle of LQR stabilization in Section 2, we adapt in Section 3 an existing robust stability certificate to systems with entry-wise uncertainty bounds on their Jacobians. In Section 4 , we present stability certificates for systems with entry-wise bounds on their Hessians, and in Section 5, we propose an algorithm adapting robust certificates to systems with varying derivatives. In Section 6, we extend the methods to the trajectory tracking problem. Finally in Section 7, we compare the robust certificates, as well as those provided by SOS programming, on numerical examples of various dimensions. An implementation is available online.

\section{Preliminaries}

We consider a nonlinear time-invariant control system:

$$
\dot{x}=f(x, u),
$$

where $x \in \mathbb{R}^{d}, u \in \mathbb{R}^{m}$, with $d, m \geq 1$. Assume there exists an equilibrium, without loss of generality at $\left(x_{0}, u_{0}\right)=(0,0)$, that is $f(0,0)=0$, and that $f$ is differentiable at the origin:

$$
f(x, u)=\underbrace{\left.\frac{\partial f}{\partial x}\right|_{0,0}}_{A} x+\underbrace{\left.\frac{\partial f}{\partial u}\right|_{0,0}}_{B} u+o(x)+o(u) .
$$

We assume that the pair $(A, B)$ is controllable. For $Q \succeq 0, R \succ 0$ symmetric matrices respectively of size $d \times d$ and $m \times m$, we define the infinite-horizon LQR cost [Lib11]:

$$
J(x):=\int_{0}^{+\infty}\left(x^{\top}(t) Q x(t)+u^{\top}(t) R u(t)\right) d t, \text { with } x(0)=x .
$$

The cost-minimizing controller is known to be:

$$
u(x)=-R^{-1} B^{\top} S x=:-K x,
$$

where $S$ is the symmetric positive definite solution of the algebraic Riccati equation (ARE), which exists because $(A, B)$ is controllable:

$$
A^{\top} S+S A-S B R^{-1} B^{\top} S=-Q .
$$

Under the closed-loop controller $u(t)=-K x(t)$, the system is autonomous with closed-loop dynamics:

$$
\dot{x}=f(x,-K x)=: g(x) \text {. }
$$

In addition, the optimal cost-to-go $V(x):=x^{\top} S x$ is used as a Lyapunov function of the nonlinear system. $V$ is a Lyapunov function over a region $\mathcal{R} \subset \mathbb{R}^{d}$ around 0 , if $V(0)=0, V(x)>0$ in $\mathcal{R} \backslash\{0\}$, and $\dot{V}(x)<0$ in $\mathcal{R}$ [SL91]. This certifies that the sublevel sets of $V$ that are included in $\mathcal{R}$ belong to the ROA of the equilibrium point: every trajectory beginning in this set will asymptotically stabilize to 0 . In practice, it is convenient to choose $\mathcal{R}$ as a sublevel set of $V$.

If the dynamics were exactly linear, then one would have:

$$
\begin{aligned}
\dot{V}(x) & =\nabla V(x) \cdot g(x)=2 x^{\top} S g(x)=x^{\top}\left(S A+A^{\top} S-2 S B K\right) x \\
& =x^{\top}\left(-Q-S B R^{-1} B^{\top} S\right) x<0, \forall x \neq 0 .
\end{aligned}
$$

Hence in the linear case, the ROA is the whole state space. In this work we will consider variations of this situation, and see how defects of linearity will affect this statement.

\section{First-Order Robustness}

In this section, we present a robust stability certificate that holds for the class of systems whose Jacobian matrix is bounded by a known quantity.

A linear differential inclusion (LDI) [AC84] is the following set-valued control problem:

$$
\dot{x} \in \Omega x, \quad x(0)=x_{0},
$$

where $\Omega$ is a convex subset of $\mathbb{R}^{d \times d}$, and $\Omega x:=\{A x, A \in \Omega\}$. The asymptotic stability of any dynamical system belonging to the LDI can be expressed as a linear matrix inequality (LMI) for some specific choices of $\Omega$ [BEGFB94]. In particular, for $\Omega=\left\{A_{0}\right\}$ we get a linear system, for $\Omega=\operatorname{Conv}\left(A_{1}, \ldots, A_{L}\right)$ a polytopic LDI (PLDI). Let $C, \Delta$ and $E$ be matrices of compatible dimensions and $\|$.$\| a matrix norm, then$ 
$\Omega=\left\{A_{0}+C \Delta E \mid\|\Delta\| \leq 1\right\}$ represents a norm-bound LDI (NLDI); if in addition $\Delta$ must be diagonal, we get a diagonal NLDI (DNLDI).

The asymptotic stability of an LDI around 0 , i.e., all initial conditions converge to 0 , can be certified by a Lyapunov function of the form $V(x)=x^{\top} P x$. This amounts to finding:

$$
P \succ 0 \text { such that } A^{\top} P+P A \prec 0, \forall A \in \Omega .
$$

This problem reduces to an LMI and is classically solved by interior-point methods [NN94] for general choices of $\Omega$. For example, in a PLDI, it reduces to a finitely constrained LMI, with one constraint at each of the vertices of the polytope $\Omega$.

LDIs are used to model uncertainty in linear systems. Any differentiable dynamical system with an equilibrium at the origin and with bounded Jacobian (including the closed loop system in equation (5p) belongs to a suitable LDI, written as an uncertain linear system:

$$
\dot{x}=A(x) x, \quad A(x) \in \Omega .
$$

$\Omega$ is a convex set of matrices that accounts for nonlinearities, uncertainties or time-variations of the dynamics. In particular, $\Omega$ can bound the deviation of a nonlinear system $\dot{x}=g(x)$ from its linearization $\dot{x}=J_{g}(0) x$. This is similar to the problem considered in [TP07], except that the perturbations lie in a closed convex set instead of a semialgebraic set.

For $i, j \in\{1, \ldots, d\}, x \in \mathbb{R}^{d}$, let $\delta_{i j}(x):=\left|A(x)_{i j}-\left(A_{0}\right)_{i j}\right|$, the entrywise deviations of the Jacobian of the dynamics from a given matrix $A_{0}$. Suppose we are given individual upper bounds on each deviation:

$$
\forall i, j \in\{1, \ldots, d\}, \quad v_{i j}:=\sup _{x \in \mathbb{R}^{d}}\left|\delta_{i j}(x)\right|,
$$

such bounds can be computed in closed form in some simple cases, or estimated by sampling, as will be discussed in Section 5.2 Stability is readily studied [BEGFB94] if $\Omega$ is a convex hull (PLDI) or a matrix ball (NLDI), which we now specify for our problem. Entrywise bounds can be fitted in both settings. Yet the description of the corresponding PLDI is intractable in large dimension: the number of vertices required to describe $\Omega$ scales as $2^{d}$.

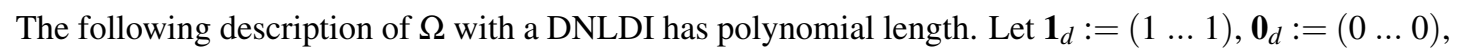

$$
\begin{gathered}
\Delta:=\operatorname{Diag}\left(\frac{\delta_{11}}{v_{11}}, \ldots, \frac{\delta_{1 d}}{v_{1 d}}, \ldots, \frac{\delta_{d 1}}{v_{d 1}}, \ldots, \frac{\delta_{d d}}{v_{d d}}\right) \in \mathbb{R}^{d^{2} \times d^{2}}, \\
C:=\left[\begin{array}{lll}
\mathbf{1}_{d} & \mathbf{0}_{d} \\
\mathbf{0}_{d} & \ddots & \\
& & \mathbf{1}_{d}
\end{array}\right]=I_{d} \otimes \mathbf{1}_{d} \in \mathbb{R}^{d \times d^{2}},
\end{gathered}
$$

$E:=\left[\begin{array}{lll}E_{1} & \ldots & E_{d}\end{array}\right]^{\top} \in \mathbb{R}^{d^{2} \times d}$, with $E_{i}=\operatorname{Diag}\left(v_{i 1}, \ldots, v_{i d}\right)$. Hence $\|\Delta\|_{2}=\sqrt{\lambda_{\max }\left(\Delta^{\top} \Delta\right)}=\sigma_{\max }(\Delta) \leq 1$, and the system belongs to the DNLDI defined by

$$
\Omega=\left\{A_{0}+C \Delta E \mid\|\Delta\| \leq 1, \Delta \text { diagonal }\right\} .
$$

Checking the asymptotic stability of a DNLDI is an LMI feasibility problem derived by applying the Sprocedure [BEGFB94]:

Proposition 1. Let $\dot{x}=A(x) x$ an uncertain linear system with entrywise bounded Jacobian. A sufficient condition for its global asymptotic stability at $O$ is the feasibility of the following LMI, for $A_{0}, C, E$ defined as above:

$$
\begin{gathered}
\text { Find } P \succ 0 \in \mathbb{R}^{d \times d}, \Lambda \succeq 0 \in \mathbb{R}^{d^{2} \times d^{2}} \text { diagonal such that: } \\
\left.\qquad \begin{array}{cc}
A_{0}^{\top} P+P A_{0}+E^{\top} \Lambda E & P C \\
C^{\top} P & -\Lambda
\end{array}\right] \prec 0 .
\end{gathered}
$$

One may optimize both $P$ and $\Lambda$ to obtain a Lyapunov function, or use a fixed predefined value of $P, e . g$, $S$ from the LQR, to check if $V(x)=x^{\top} P x$ is a valid Lyapunov function.

\section{SECOND-ORdER Robustness}

Let us derive robust stability certificates like in the previous section, except that now, they hold for a class of systems whose Hessian tensor is bounded by a known quantity. 
4.1. Condition on the Sublevel Sets. Let $\varphi: \mathbb{R}^{d} \rightarrow \mathbb{R}^{d}$, such that each $\varphi_{k}$ is twice continuously differentiable with bounded Hessian on a closed ball $\mathcal{B}$ centered around 0 . Then, using Taylor's formula, for any $x \in \mathbb{R}^{d}$, there exists a symmetric matrix $H^{k}(x)$ such that for all $k \in\{1, \ldots, d\}$ :

$$
\begin{gathered}
\varphi_{k}(x)=\varphi_{k}(0)+\nabla \varphi_{k}(0)^{\top} x+\frac{1}{2} x^{\top} H^{k}(x) x, \\
\text { with } H_{i j}^{k}(x)=2 \int_{0}^{1}(1-t) \frac{\partial^{2} \varphi_{k}}{\partial x_{i} \partial x_{j}}(t x) \mathrm{d} t .
\end{gathered}
$$

Hence $\forall i, j, k, \forall x \in \mathcal{B},\left|H_{i j}^{k}(x)\right| \leq \max _{y \in \mathcal{B}}\left|\frac{\partial^{2} \varphi_{k}}{\partial x_{i} \partial x_{j}}(y)\right|$. Note that this is also true if $\mathcal{B}$ is an ellipsoid around 0 . This applies to the function $g$ of Eq. (5):

$$
g_{k}(x)=f_{k}(x,-K x)=(A-B K)_{k} \cdot x+\frac{1}{2} x^{\top} H^{k}(x) x,
$$

where $X_{k}$. denotes the $k$-th row-vector of a matrix $X$. The time derivative of the candidate Lyapunov function is:

$$
\begin{aligned}
\dot{V}(x) & =2 x^{\top} S\left((A-B K) x+\frac{1}{2}\left(x^{\top} H^{k}(x) x\right)_{k \in\{1, \ldots, d\}}\right) \\
& =x^{\top}\left(-Q-S B R^{-1} B^{\top} S+\sum_{k=1}^{d}\left(S_{k} \cdot x\right) H^{k}(x)\right) x .
\end{aligned}
$$

Let $\mathcal{B}_{\rho}:=\left\{x \mid x^{\top} S x \leq \rho\right\}$ for $\rho>0$, a sublevel set of $V$. A sufficient condition for $\mathcal{B}_{\rho}$ to be an ROA around 0 is that $-Q-S B R^{-1} B^{\top} S+\sum_{k}\left(S_{k} \cdot x\right) H^{k}(x) \prec 0$ for all $x \in \mathcal{B}_{\rho}$. Let $M:=Q+S B R^{-1} B^{\top} S \succ 0$, the condition is equivalent to:

$$
\forall x \in \mathcal{B}_{\rho}, \quad \sum_{k=1}^{d}\left(S_{k} . x\right) \tilde{H}^{k}(x) \prec I_{d},
$$

where $\tilde{H}^{k}(x):=M^{-1 / 2} H^{k}(x) M^{-1 / 2}$. We denote by $\tilde{H}(x)$ the tensor composed of the matrices $\tilde{H}^{k}(x)$, for $k \in\{1, \ldots, d\}$.

The goal is to find the largest $\rho$ such that condition 8 holds, which will in turn prove that $\mathcal{B}_{\rho}$ is an ROA around 0 . To simplify this problem, we will decouple the two dependencies in $x$. On the one hand, the contribution of $S_{k} . x$ will be bounded by two different bounds that we present below. On the other hand, the tensor $\tilde{H}(x)$ is bounded globally, independently from $\rho$. Of course, this is not always possible in general, and a tighter analysis of the Hessian with local bounds depending on $\rho$ will be discussed in Section 5 For now, assume that we are given an oracle on the magnitude $e_{i}^{\top} \tilde{H}^{k}(x) e_{j}\left(e_{i}\right.$ being the $i$-th unit vector) of $\tilde{H}(x)$ along $d^{2}$ directions for each matrix $\tilde{H}^{k}(x)$, of the form:

$$
\forall x, \tilde{H}(x) \in \Xi:=\left\{T \in \mathbb{R}^{d^{3}}|\forall i, j, k,| T_{i j}^{k} \mid \leq u_{i j}^{k},\left(T^{k}\right)^{\top}=T^{k}\right\},
$$

for some $d \times d \times d$ tensor $U$ of nonnegative real numbers $u_{i j}^{k}$, with $\left(U^{k}\right)^{\top}=U$ for all $k \in\{1, \ldots, d\}$.

A relaxation of condition $(8)$ is then:

$$
\sup _{x^{\top} S x \leq \rho} \sup _{T \in \Xi} \lambda_{\max }\left(\sum_{k=1}^{d}\left(S_{k} \cdot x\right) T^{k}\right)<1 .
$$

With a simple change of variable and rescaling, the largest $\rho$ fulfilling the above condition is then given by:

$$
\rho=\frac{1}{\lambda^{2}}, \text { where } \lambda:=\sup _{\|y\|_{2} \leq 1} \sup _{T \in \Xi} \lambda_{\max }\left(\sum_{k=1}^{d}\left(S_{k}^{1 / 2} y\right) T^{k}\right) .
$$

4.2. Two Upper Bounds on $\lambda$. The first bound is based on the following fact:

$$
\sup _{\|y\|_{2} \leq 1} \sup _{T \in \Xi}\left\|\sum_{k=1}^{d}\left(S_{k}^{1 / 2} y\right) T^{k}\right\|_{2} \leq \sup _{\|y\|_{2} \leq 1} \sum_{k=1}^{d}\left|S_{k}^{1 / 2} y\right| \sup _{T^{k} \in \Xi^{k}}\left\|T^{k}\right\|_{2},
$$

where $\Xi^{k}$ is the projection of $\Xi$ onto its $k$-th coordinate subspace. Let $Z$ be a matrix with rows $Z_{k}$. := $\left(\sup _{T \in \Xi^{k}}\left\|T^{k}\right\|_{2}\right) S_{k}^{1 / 2}$,

$$
\lambda \leq \sup _{\|y\|_{2} \leq 1}\|Z y\|_{1} \leq \sqrt{d} \sup _{\|y\|_{2} \leq 1}\|Z y\|_{2}=\sqrt{d}\|Z\|_{2}
$$


where $\|\cdot\|_{2}$ denotes both the Euclidean norm and the corresponding matrix induced norm. With equation 10, this guarantees that $\mathcal{B}_{\rho_{b}}$ is an ROA for

$$
\rho_{b}:=\frac{1}{d\left\|D S^{1 / 2}\right\|_{2}^{2}}, \text { with } D=\operatorname{Diag}\left(\left(\sup _{T^{k} \in \Xi^{k}}\left\|T^{k}\right\|_{2}\right)_{k}\right) .
$$

The following lemma explains how to compute the entries of $D$.

Lemma 1. Let $V$ be a nonnegative symmetric $d \times d$ matrix with entries $\left(v_{i j}\right)$. Let $\xi$ be the set of symmetric $d \times d$ matrices $A$ such that for all $i, j \in\{1, \ldots, d\},\left|A_{i j}\right| \leq v_{i j}$. Then:

$$
\max _{A \in \xi}\|A\|_{2}=\|V\|_{2} \text {. }
$$

Proof. Since $\xi$ is centered around 0, we only look for the largest eigenvalue:

$$
\sup _{\|x\|_{2} \leq 1, A \in \xi} x^{\top} A x=\sup _{\|x\|_{2} \leq 1} \sup _{A \in \xi} \sum_{i} a_{i i} x_{i}^{2}+2 \sum_{i<j} a_{i j} x_{i} x_{j} .
$$

Maximizing with respect to $A$, we get $a_{i i}=v_{i i}$, and

$$
\forall i<j, \quad a_{i j}= \begin{cases}v_{i j} & \text { if } x_{i} x_{j} \geq 0 \\ -v_{i j} & \text { else. }\end{cases}
$$

And then, $x^{\top} A x=\sum_{i} v_{i i} x_{i}^{2}+2 \sum_{i<j} v_{i j}\left|x_{i} x_{j}\right|=|x|^{\top} V|x|$. The full problem becomes:

$$
\sup _{\|x\|_{2} \leq 1, x \succeq 0} x^{\top} V x .
$$

The Perron-Frobenius theorem ensures that for any nonnegative square matrix, there exists a nonnegative real eigenvalue with at least one nonnegative eigenvector. Any other eigenvalue's modulus is smaller than this eigenvalue. $V$ being symmetric, all its eigenvalues are real, hence the result.

Remark: If the bounds on the entries of $A$ are not centered around 0 , it is possible to write $A=A^{\prime}+\bar{A}$, where the entries of $\bar{A}$ are symmetrically bounded, and $\|A\|_{2} \leq\left\|A^{\prime}\right\|_{2}+\|\bar{A}\|_{2}$.

The lemma leads to another bound on $\lambda$. If $T \in \Xi$ :

$$
\forall i, j, k,\left|\sum_{k}\left(S_{k}^{1 / 2} y\right) T_{i j}^{k}\right| \leq \sum_{k}\left|S_{k}^{1 / 2} y\right| u_{i j}^{k} \leq \sum_{k}\left\|S_{k .}^{1 / 2}\right\| \cdot\|y\| u_{i j}^{k}
$$

Applying the previous result to the matrix whose entries are the middle term in the inequality above yields:

$$
\lambda \leq \lambda_{\max }\left(\sum_{k} \sqrt{S_{k} \cdot S^{-1} S_{k}^{\top}} U^{k}\right)=: \lambda_{a} .
$$

The following theorem states the two stability certificates derived above.

Theorem 1. Consider the control system and the matrices $S, K$ and $M$ defined in Sections 2 and 4 Assume that the closed-loop system $x \mapsto g(x)=f(x,-K x)$ is twice continuously differentiable and the following condition holds:

$$
\forall x \in \mathbb{R}^{d}, \forall i, j, k \in\{1, \ldots, d\},\left|\left[M^{-1 / 2} \nabla^{2} g_{k}(x) M^{-1 / 2}\right]_{i j}\right| \leq u_{i j}^{k},
$$

where for each $k$, the matrix $U^{k}$ with entries $\left(u_{i j}^{k}\right)_{i j}$ is symmetric and nonnegative. Then $\mathcal{B}_{\rho_{a}}$ and $\mathcal{B}_{\rho_{b}}$ are two ROAs of the closed-loop system, for $\rho_{a}=1 / \lambda_{a}^{2}, \rho_{b}=1 / \lambda_{b}^{2}$ and

$$
\begin{gathered}
\lambda_{a}=\lambda_{\max }\left(\sum_{k} \sqrt{S_{k} \cdot S^{-1} S_{k}^{\top}} U^{k}\right), \\
\lambda_{b}=\sqrt{d}\left\|D S^{-1 / 2}\right\|_{2}, \text { with } D=\operatorname{Diag}\left(\left\|U^{k}\right\|_{2}\right)_{k} .
\end{gathered}
$$

This theorem provides certified ROAs from a given Lyapunov function $V(x)=x^{\top} S x$, which comes from the LQR. The elliptical shape of the ROAs is fixed, as opposed to Proposition 1, where the Lyapunov function $x^{\top} P x$ can be optimized. In the rest of the paper, we will consider $P$ to be fixed in the LMI (7). There does not seem to be a straightforward extension of our second-order certificates with an optimized Lyapunov function. 


\section{Iterative Algorithm}

5.1. Stability Certificates. The bounds introduced in Sections 3 and 4 readily give robust stability certificates that hold for a whole class of dynamics with suitably bounded derivatives. It is also possible to apply the same methods to a single known dynamics, if its derivatives can be bounded efficiently. In general the bounds on the derivatives depend on where they are computed: the larger the region, the larger the bounds. But such bounds must be computed on a sufficiently large region containing the sublevel set where the system stability is asserted.

With the notations of the two previous sections, and given equations $\left(\begin{array}{ll|l|l|}7 & 11\end{array}\right.$, we define three stability certificates:

$$
\begin{aligned}
\mathcal{C}_{1}:\left(S, \rho_{u p}, \Omega\right) & \rightarrow \rho_{u p} \mathbf{1}_{\mathrm{LMI}} \text { (7) is feasible } \\
\mathcal{C}_{2}^{a, b}:\left(S, \rho_{u p}, \Xi\right) & \rightarrow \min \left(\rho_{a, b}, \rho_{u p}\right),
\end{aligned}
$$

meaning that $\left\{x^{\top} S x \leq \mathcal{C}_{1}\left(S, \rho_{u p}, \Omega\right)\right\}$ is an ROA if the derivatives of the dynamics are bounded by $\Omega$ (resp. $\Xi$ ) and if $\rho_{u p}$ is an upper bound on $\rho$ used to compute $\Omega$ (resp. $\Xi$ ).

5.2. Oracle on the Derivatives. Suppose we have an oracle $\mathcal{O}$ computing, on a domain $\mathcal{D}$, a bound $\mathcal{O}(\mathcal{D})$ on the derivatives, corresponding to sets $\Omega$ or $\Xi$ above. Our methods compute $\rho=\mathcal{C}\left(S, \rho_{u p}, \mathcal{O}(\mathcal{D})\right)$. Then $\mathcal{B}_{\rho}$ is an ROA if the whole trajectory to 0 stays inside $\mathcal{D}$ (else the assumptions on the derivatives would be violated). A simple way to ensure that is to choose $\mathcal{D}$ as a sublevel set of $V$ containing $\mathcal{B}_{\rho}$, i.e., $\mathcal{B}_{\rho_{u p}}$ for $\rho_{u p} \geq \rho$.

For a quadratic dynamical system, each entry of the Hessian is constant and each entry of the Jacobian is an affine function. For any $c \in \mathbb{R}^{d}$, the supremum of a linear function $x \mapsto c^{\top} x$ on an ellipsoid can be computed in closed-form, as follows:

$$
\sup _{x^{\top} S x \leq \rho_{\text {up }}} c^{\top} x=\sqrt{\rho_{u p}}\left\|S^{-1 / 2} c\right\|_{2} .
$$

For a third-order polynomial system, the entries of its Hessian are affine, hence the previous formula can be applied, and those of the Jacobian are polynomials of degree two. The following formula gives an exact upper bound for a quadratic monomial over an ellipsoid and naturally extends to polynomials of degree two after an affine change of variable:

$$
\sup _{x^{\top} S x \leq \rho_{u p}} x^{\top} J x=\rho_{u p} \lambda_{\max }\left(S^{-1 / 2} J S^{-1 / 2}\right) .
$$

In large dimension, manually identifying each coefficient of the derivatives of a second or third order polynomial dynamics might be tedious: the Hessian tensor indeed contains $d^{3}$ entries. One solution is to define the polynomial dynamics $f$ with symbolic expressions and to obtain the derivatives with a computed algebra system. Another one is to sample derivatives at a few but different points with automatic differentiation [ $\left[\mathrm{PGM}^{+} 19\right]$, to fit a low order polynomial model, and then to maximize it in closed form.

For generic dynamics, one can sample derivatives, e.g., by automatic differentiation, using analytical derivatives [CM18a] for rigid body dynamics, or from direct physical measurements on the system. Of course, bounding the samples only provides lower-estimates of the oracle, possibly resulting in overoptimistic stability certificates. Hence extra caution must be taken to ensure sufficient precision of the oracles. In particular, samples can be collected offline or in parallel in order to mitigate the computation times. Besides, maximization by sampling suffers from the curse of dimensionality, yet efficiency improvements can be expected with Bayesian optimization [Moc12] or other global optimization tools.

5.3. Algorithm. A simple ROA estimation algorithm (see Algorithm 1) consists in iteratively bounding the derivatives and producing stability certificates, i.e., alternating calls of $\mathcal{O}$ and $\mathcal{C}$. $\rho_{0}$ is an initial upper bound on the size of the ROA. Each step of the loop provides a certificate that $\mathcal{B}_{\rho}$ is an ROA, and this region grows at each iteration, the sequence of $\rho$ s being nondecreasing. The number of iterations before the algorithm stops depends on both the initial guess $\rho_{0}$ and the step size $\eta$. In our experiments, we typically require from 10 up to 20 iterations.

\section{Trajectory TraCking}

The certificates and the algorithm presented in the previous sections are applied around the equilibrium point of a dynamical system. They can be extended to the more general problem of trajectory tracking, as described hereafter. 


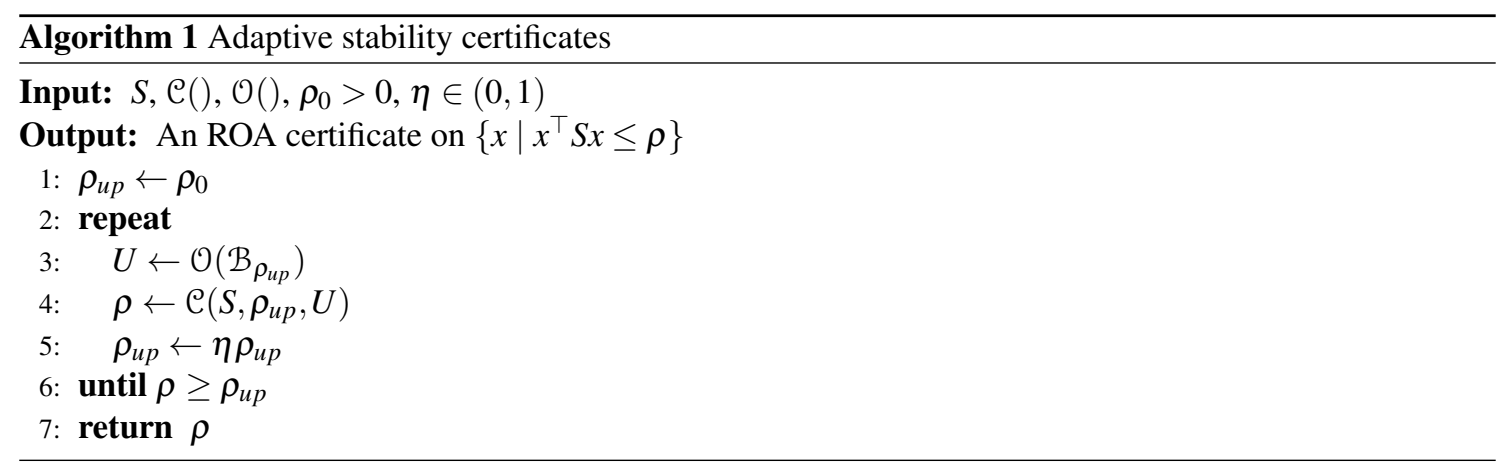

Let $\left(x_{0}(t), u_{0}(t)\right)$, for $t \in\left[0, t_{f}\right]$ be a reference trajectory with final state $x_{f}:=x_{0}\left(t_{f}\right)$. For a nearby trajectory $(x(t), u(t))$, let $\bar{x}(t):=x(t)-x_{0}(t), \bar{u}(t):=u(t)-u_{0}(t)$. The linearized dynamics reads:

$$
\dot{\bar{x}}(t)=A(t) \bar{x}(t)+B(t) \bar{u}(t)+o(\bar{x}(t))+o(\bar{u}(t)) .
$$

Let $\mathcal{B}_{f}$ a target region $\left\{x \mid\left(x-x_{f}\right)^{\top} S_{f}\left(x-x_{f}\right) \leq 1\right\}$, for some $S_{f} \succeq 0$. We define the finite-horizon LQR problem [Lib11] with the following tracking cost:

$$
\int_{0}^{t_{f}}\left(\bar{x}(t)^{\top} Q \bar{x}(t)+\bar{u}(t)^{\top} R \bar{u}(t)\right) \mathrm{d} t+\bar{x}^{\top}\left(t_{f}\right) S_{f} \bar{x}\left(t_{f}\right) .
$$

For $t \in\left[0, t_{f}\right]$, the optimal cost-to-go is $V(x, t)=\bar{x}^{\top} S(t) \bar{x}, S(t)$ being the solution of the Riccati differential algebraic equation (RDE):

$$
\dot{S}=-Q+S B R^{-1} B^{\top} S-S A-A^{\top} S, \quad S\left(t_{f}\right)=S_{f},
$$

with controller $\bar{u}(t)=-K(t) \bar{x}(t):=-R^{-1} B^{\top}(t) S(t) \bar{x}(t)$.

We want to estimate the time-varying region (also called "funnel" [TMTR10]) $\mathcal{B}(t):=\left\{x \mid F(x, t) \in \mathcal{B}_{f}\right\}$, where $F(x, t)$ is the integrated closed-loop dynamics with control $u($.$) from t$ to $t_{f}$. In particular, $\mathcal{B}\left(t_{f}\right)=\mathcal{B}_{f}$. $\mathcal{B}(t)$ is a region where applying $u(t)=u_{0}(t)+\bar{u}(t)$ will make the trajectory reach $\mathcal{B}\left(t_{f}\right)$ after time $t_{f}$. If in addition $\mathcal{B}\left(t_{f}\right)$ is included in an ROA around 0 , the trajectory will finally reach 0 in finite time.

We consider regions $\mathcal{B}(t):=\{x \mid 0 \leq V(x, t) \leq \rho(t)\}$. A sufficient condition for $\mathcal{B}(t)$ to be a funnel is [TMT11]:

$$
V(x, t) \geq 0, \forall x \in \mathcal{B}(t) \text { and } \dot{V}(x, t) \leq \dot{\rho}(t), \forall x \in \partial \mathcal{B}(t) .
$$

We drop some occurrences of the time variable $t$ to simplify the notations. $S(t)$ being a positive definite matrix [Lib11] for any $t \in\left[0, t_{f}\right]$, the first condition holds, the second one is:

$$
\dot{V}(x, t)=2 \bar{x}^{\top} S \dot{\bar{x}}+\bar{x}^{\top} \dot{S} \bar{x} \leq \dot{\rho}, \forall x \in\left\{x \mid \bar{x}^{\top} S \bar{x}=\rho\right\} .
$$

If the closed-loop system is an LDI $\dot{\bar{x}}=\tilde{A}(t, x) \bar{x}$ in $\left\{x \mid \bar{x}^{\top} S \bar{x}=\rho\right\}$, with $\tilde{A} \in \Omega(\rho)$, a sufficient condition is:

$$
\forall \tilde{A} \in \Omega(\rho), \quad \tilde{A}^{\top} S+S \tilde{A}+\dot{S}-\frac{\dot{\rho}}{\rho} S \preceq 0 .
$$

This can be fit into the LDI framework presented in Section 3 , just by shifting the set $\Omega(\rho)$ to the set

$$
\tilde{\Omega}(\rho, \dot{\rho}):=\left\{\tilde{A}+\frac{1}{2} S^{-1} \dot{S}-\frac{1}{2} \frac{\dot{\rho}}{\rho} I_{d} \mid \tilde{A} \in \Omega(\rho)\right\} .
$$

Now if the closed-loop system is known up to order two in $\left\{x \mid \bar{x}^{\top} S \bar{x}=\rho\right\}$, say $\dot{\bar{x}}=(A-B K) \bar{x}+$ $\frac{1}{2} \bar{x}^{\top} H(t, x) \bar{x}$, with $H(t, x) \in \Xi(\rho)$. Using that $S($.$) is a solution of equation [15$, we obtain the following sufficient condition: $\forall y$ such that $\|y\|_{2}=1, \forall H \in \Xi(\rho)$,

$$
-Q-S B R^{-1} B^{\top} S-\frac{\dot{\rho}}{\rho} S+\sqrt{\rho} \sum_{k=1}^{d}\left(S_{k}^{1 / 2} y\right) H^{k} \preceq 0 .
$$

If $N(\rho, \dot{\rho})=Q+S B R^{-1} B^{\top} S+\frac{\dot{\rho}}{\rho} S \succ 0$, let $\tilde{\Xi}(\rho, \dot{\rho})$ the shifted set $\left\{N^{-1 / 2} H N^{-1 / 2} \mid H \in \Xi(\rho)\right\}$, we must check that:

$$
\forall y \text { s.t. }\|y\|_{2}=1, \forall \tilde{H} \in \tilde{\Xi}(\rho, \dot{\rho}), \sqrt{\rho} \sum_{k=1}^{d}\left(S_{k}^{1 / 2} y\right) \tilde{H}^{k} \preceq I_{d} \text {. }
$$


Under such conditions, $\rho($.$) is built backwards in time through backward integration, beginning with$ $\rho\left(t_{f}\right)=1$. At each time step, given $\rho$, a greedy strategy is to choose $\dot{\rho}$ as the smallest possible value such that the sufficient condition is enforced. Since $\rho($.$) is computed backwards, this maximizes \rho(t-\mathrm{d} t)$, hence locally the funnel's volume. Also, for both first and second order cases, the sufficient condition is monotonically more restrictive as $\dot{\rho}$ decreases. A simple algorithm is to start with a large positive $\dot{\rho}$, compute the set $\tilde{\Omega}(\rho, \dot{\rho})$ or $\tilde{\Xi}(\rho, \dot{\rho})$, check that the sufficient condition holds, and progressively decrease $\dot{\rho}$ until it no longer does (possibly with $\dot{\rho}<0$ if $N \succ 0$ is still enforced, when applicable).

\section{NUMERICAL EXPERIMENTS}

7.1. Definition of the Systems and Implementation Details. The code to reproduce the experiments is available online ${ }^{1}$ The first two systems, an electrical oscillator and a floating satellite with commanded torques, are taken from the Matlab material of [TMT11]. The third one is an underactuated double pendulum, with the actuated joint between the two arms (also called "acrobot" in [Sut96]). The last one corresponds to the UR5 robotic arm from Universal Robots ${ }^{2}$, with 6 actuated joints. The dynamics of these dynamical systems are described hereafter.

Vanderpol. $d=2, m=0$ (unactuated), $x_{0}=\mathbf{0}_{2}^{\top}, Q=I_{2}$. The dynamics is a polynomial of degree 3 :

$$
\forall x=\left(x_{1}, x_{2}\right) \in \mathbb{R}^{2}, \quad f(x)=\left(-x_{2}, x_{1}+x_{2}\left(x_{1}^{2}-1\right)\right)^{\top} .
$$

Satellite. $d=6, m=3,\left(x_{0}, u_{0}\right)=\left(\mathbf{0}_{6}^{\top}, \mathbf{0}_{3}^{\top}\right), Q=I_{6}, R=10 \times I_{3}$, the dynamics is a polynomial of degree 3 . Let $J=\operatorname{Diag}(5,3,2)$. For $x=\left(\omega^{\top}, \sigma^{\top}\right)^{\top} \in \mathbb{R}^{6}$, with $\omega, \sigma \in \mathbb{R}^{3}, f(x, u)=\left(\dot{\omega}^{\top}, \dot{\sigma}^{\top}\right)^{\top}$,

$$
\begin{aligned}
& \dot{\omega}=J^{-1}(u-\omega \times J \omega) \\
& \dot{\sigma}=\frac{1}{4}\left(\left(1-\|\sigma\|^{2}\right) I_{3}+2 \sigma \sigma^{\top}-2\left[\begin{array}{ccc}
0 & \sigma_{3} & \sigma_{2} \\
\sigma_{3} & 0 & \sigma_{1} \\
\sigma_{2} & \sigma_{1} & 0
\end{array}\right]\right) \omega .
\end{aligned}
$$

Pendulum. $d=4, m=1, u_{0}=0, x_{0}=\mathbf{0}_{4}^{\top}$ (bottom) or $x_{0}=(\pi, \pi, 0,0)^{\top}$ (top), $Q=I_{4}, R=1$. Let $g=9.8$, $\ell=0.5$ and $\mu=1$. For $x=\left(\theta_{1}, \theta_{2}, p_{1}, p_{2}\right)^{\top}, f(x, u)$ is defined by:

$$
\begin{aligned}
& \dot{\theta}_{1}=\frac{6}{\mu \ell^{2}} \frac{2 p_{1}-3 \cos \left(\theta_{1}-\theta_{2}\right) p_{2}}{16-9 \cos ^{2}\left(\theta_{1}-\theta_{2}\right)} \\
& \dot{\theta}_{2}=\frac{6}{\mu \ell^{2}} \frac{8 p_{2}-3 \cos \left(\theta_{1}-\theta_{2}\right) p_{1}}{16-9 \cos ^{2}\left(\theta_{1}-\theta_{2}\right)} \\
& \dot{p}_{1}=-\frac{\mu \ell^{2}}{2}\left(\dot{\theta}_{1} \dot{\theta}_{2} \sin \left(\theta_{1}-\theta_{2}\right)+\frac{3 g}{\ell} \sin \theta_{1}\right) \\
& \dot{p}_{2}=-\frac{\mu \ell^{2}}{2}\left(-\dot{\theta}_{1} \dot{\theta}_{2} \sin \left(\theta_{1}-\theta_{2}\right)+\frac{g}{\ell} \sin \theta_{1}\right)+u .
\end{aligned}
$$

Robotic arm. Here $d=12, m=6, x_{0}=\left(q_{0}^{\top}, \mathbf{0}_{6}\right)^{\top}, q_{0}$ is the initial configuration $q_{0}=(0,-\pi / 5,-3 \pi / 5,0,0,0)$, $Q=I_{12}, R=I_{6} . u_{0}$ is such that $f\left(x_{0}, u_{0}\right)=0$ and is computed by the recursive Newton-Euler algorithm (RNEA) implemented in the $\mathrm{C}++$ library Pinocchio $\left[\mathrm{CSB}^{+} 19\right]$. The forward dynamics $f(x, u)$ is computed via the articulated body algorithm (ABA).

The software used for the SOS based certificates is adapted from the Matlab material of [TMT11]. The oracles on the derivatives are computed either in closed form, for Vanderpol and the Hessian of Satellite, using formulas (13) and (14), or by sampling $p$ derivatives. Using automatic differentiation in PyTorch [ $\left.\mathrm{PGM}^{+} 19\right]$, we sample $p=10^{4}$ Jacobians for Satellite, $p=10^{3}$ Jacobians and Hessians for Pendulum. For Robot, $p=5 \times 10^{4}$ and the Jacobians of the dynamics are computed analytically [CM18a], and we use finite differences on the first partial derivatives to approximate the Hessians. It is important to notice at this stage that more advanced methods to efficiently compute these Hessians could improve the whole computation time of our methods, for instance by code-generating the second-order derivatives computed by automatic differentiation. Yet, the proposed solution already provides competitive timings.

7.2. Results. The performances of the certificates are compared in Table 1 , both in terms of radius of $\mathcal{B}_{\rho}$ and volume $v \propto \rho^{d / 2} / \sqrt{|S|}$, the latter exacerbating differences in large dimensions. The volume, divided by the volume of the state space, is roughly the inverse of the number of ROAs that would have covered

Www.github.com/eloiseberthier/Fast-Robust-ROA

www.universal-robots.com/products/ur5-robot 
TABLE 1. Radius and volume of the certified ROA for the different methods, relative to the values obtained by sampling for reference.

\begin{tabular}{|l|c|c|c|c|c|c|c|c|c|c|}
\hline \multirow{2}{*}{ Dynamics } & \multicolumn{2}{|c|}{$\mathcal{C}_{\mathbf{1}}$} & \multicolumn{2}{c|}{$\mathcal{C}_{\mathbf{2}}^{\mathbf{a}}$} & \multicolumn{2}{c|}{$\mathcal{C}_{\mathbf{2}}^{\mathbf{b}}$} & \multicolumn{2}{c|}{ SOS } & \multicolumn{2}{c|}{ sampling } \\
\cline { 2 - 11 } & $\rho / \rho_{s}$ & $v / v_{s}$ & $\rho / \rho_{s}$ & $v / v_{s}$ & $\rho / \rho_{s}$ & $v / v_{s}$ & $\rho / \rho_{s}$ & $v / v_{s}$ & $\rho / \rho_{s}$ & $v / v_{s}$ \\
\hline \hline Vanderpol & 0.20 & 0.20 & 0.14 & 0.14 & 0.10 & 0.10 & $\mathbf{1}$ & $\mathbf{1}$ & 1 & 1 \\
\hline Satellite & $2.9 \times 10^{-2}$ & $2.6 \times 10^{-5}$ & $9.3 \times 10^{-2}$ & $9.4 \times 10^{-4}$ & $7.9 \times 10^{-2}$ & $5.7 \times 10^{-4}$ & $\mathbf{0 . 9 3}$ & $\mathbf{0 . 8 2}$ & 1 & 1 \\
\hline Pend. (bot.) & $3.2 \times 10^{-2}$ & $1.1 \times 10^{-3}$ & $3.5 \times 10^{-2}$ & $1.2 \times 10^{-3}$ & $\mathbf{4 . 2} \times \mathbf{1 0}^{-2}$ & $\mathbf{1 . 9} \times \mathbf{1 0}^{-3}$ & $1.4 \times 10^{-2}$ & $2.0 \times 10^{-4}$ & 1 & 1 \\
\hline Pend. (top) & $5.1 \times 10^{-3}$ & $2.6 \times 10^{-5}$ & $4.5 \times 10^{-2}$ & $2.0 \times 10^{-3}$ & $\mathbf{4 . 7} \times \mathbf{1 0}^{-\mathbf{2}}$ & $\mathbf{2 . 2} \times \mathbf{1 0}^{-\mathbf{3}}$ & N.A. & N.A. & 1 & 1 \\
\hline Robot & $2.4 \times 10^{-3}$ & $1.8 \times 10^{-16}$ & $7.1 \times 10^{-3}$ & $1.5 \times 10^{-13}$ & $\mathbf{1 . 5} \times \mathbf{1 0}^{-\mathbf{2}}$ & $\mathbf{1 . 2} \times \mathbf{1 0}^{-11}$ & N.A. & N.A. & 1 & 1 \\
\hline
\end{tabular}

TABLE 2. CPU time (s) per iteration, except for SOS (total time).

\begin{tabular}{|l|c|c|c|c|}
\hline Dynamics & $\mathcal{O}+\mathcal{C}_{\mathbf{1}}$ & $\mathcal{O}+\mathcal{C}_{\mathbf{2}}^{\mathbf{a}}$ & $\mathcal{O}+\mathcal{C}_{\mathbf{2}}^{\mathbf{b}}$ & SOS \\
\hline \hline Vanderpol & $1.8 \times 10^{-3}$ & $1.1 \times 10^{-4}$ & $1.6 \times 10^{-4}$ & 0.05 \\
\hline Satellite & 1.2 & 0.17 & 0.17 & 32 \\
\hline Pend. (bot.) & 2.3 & 15 & 15 & 132 \\
\hline Robot & 2.3 & 32 & 33 & N.A. \\
\hline
\end{tabular}

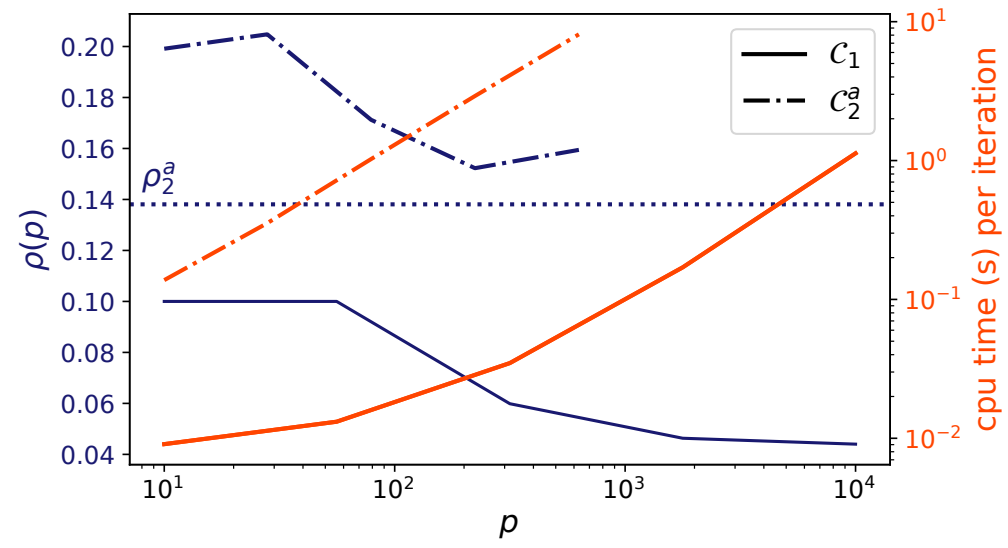

FIGURE 1. Results of $\mathcal{C}_{1}, \mathcal{C}_{2}^{a}$, and cpu time on Satellite, depending on $p$.

it. All the values in the table are divided by the ground truth $\rho_{s}$, the maximal $\rho$ such that $\forall x^{\top} S x \leq \rho$, $\dot{V}(x)<0$, estimated by sampling a very large number of points. Apart from SOS on the first two problems, all methods are very far from estimating the true maximal ROA.

For the SOS method on Pendulum, because the dynamics is non polynomial, we substitute the odd function $f$ by its Taylor expansion around the equilibrium, truncated at order $n=7$. The result is sensitive to the order: for $n=2, f$ is linear hence $\rho=+\infty$, whereas $\rho$ decreases for higher orders. It is unclear which one to choose, and the results are no longer certified. At the top position, an utterly unstable position, SOS fails to provide a positive $\rho$, regardless of $n \geq 3$.

Table 2 reports the corresponding CPU running times on a standard laptop. The code, in Python, is not optimized, except the SOS method and the LMI solver for $\mathcal{C}_{1}$ which are in Matlab. Our methods are much lighter than SOS, yet one must keep in mind that Algorithm 1 typically calls the oracle and the certificate 10 times. Nonetheless, this allows to tackle systems of larger dimensions, like Robot. If the oracle uses sampling, this dominates the running time. Figure 1 compares the running times of bounding the derivatives for $\mathcal{C}_{1}$ and $\varrho_{2}^{a}$, depending on the number of samples $p$, on Satellite. At fixed $p$, it is of course longer to sample Hessians than Jacobians. The sampling oracle overestimates $\rho$, but this tends to stabilize for reasonable values of $p$, as seen for $\rho_{2}^{a}$ which can also be computed using a closed-form oracle.

We also experiment trajectory tracking of a given reference trajectory of Vanderpol, with $x_{f}=(-1,-1)^{\top}$, $t_{f}=2$. The target region $\mathcal{B}_{f}=\left\{x \mid \bar{x}^{\top} S_{f} \bar{x} \leq 1\right\}$ is the largest ellipsoid included in $\mathcal{R}$, an ROA around 0 


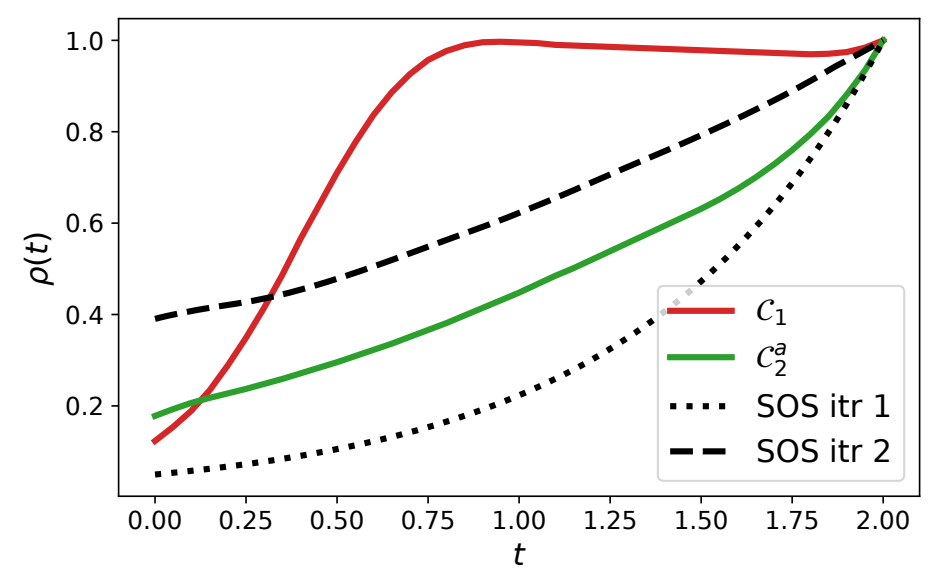

FIGURE 2. $\rho(t)$ with different certificates, around a trajectory of Vanderpol. The total CPU time is $7 \mathrm{~s}$ for two iterations of SOS, roughly 1 s for $\mathcal{C}_{1}, \mathrm{C}_{2}^{a}$.

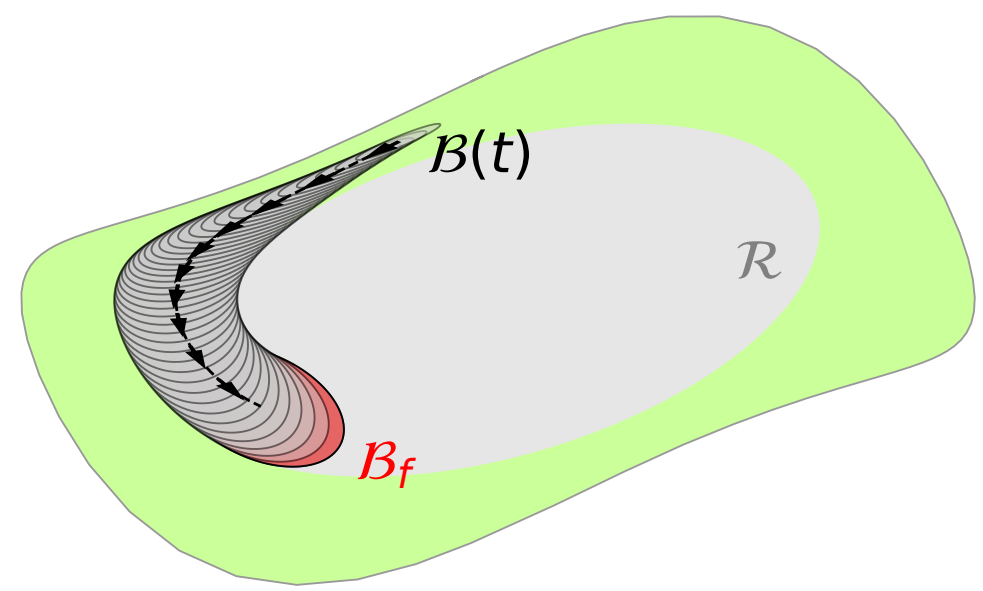

FIGURE 3. A funnel $\mathcal{B}(t)$ around a trajectory of Vanderpol, obtained with $\mathcal{C}_{1}$. The statespace is in green, $\mathcal{R}$ in light gray is an ROA around 0 , and $\mathcal{B}_{f}$ in red is the target region. The reference trajectory is displayed with arrows.

computed by SOS. In Figure 3 the state-space is in green, $\mathcal{R}$ in light gray and $\mathcal{B}_{f}$ in red. The funnel $\mathcal{B}(t)$, in gray, is computed backwards, with one or two iterations of the SOS-based algorithm of [TMT11], and with the methods of Section 6 . Figure 2 shows our certificates lead to competitive values of $\rho(t)$, with faster computations.

\section{Conclusion}

The stability certificates presented in this paper are both fast to compute, and robust over a class of bounded-derivatives dynamics. They readily extend to the trajectory tracking problem, with a linear complexity in the number of time steps. Such certificates can be easily implemented and enable handling non-polynomial, large dimensional control systems that were previously out of reach. The complexity is transferred from the certificate to a derivative-bounding oracle, which can be estimated efficiently in some cases, including rigid body dynamic systems in robotics. The certificates for trajectory tracking can in turn be integrated into the LQR-trees framework for global motion planning. They are more conservative than competing methods, yet faster, hence repeating calls to these certificates around numerous different trajectories, as done in the LQR-trees algorithm, could be more efficient overall. Providing empirical evidence or counter-evidence for this trade-off phenomenon in real-world control systems would be an interesting avenue for future research. 


\section{ACKNOWLEDGEMENTS}

This work was supported by the Direction Générale de l'Armement, and by the French government under management of Agence Nationale de la Recherche as part of the "Investissements d'avenir" program, reference ANR-19-P3IA-0001 (PRAIRIE 3IA Institute).

\section{REFERENCES}

[AC84] Jean-Pierre Aubin and Arrigo Cellina, Differential inclusions: Set-valued maps and viability theory, Springer, 1984.

[BEGFB94] Stephen Boyd, Laurent El Ghaoui, Eric Feron, and Venkataramanan Balakrishnan, Linear matrix inequalities in system and control theory, vol. 15, Siam, 1994.

[Che04] Graziano Chesi, Estimating the domain of attraction for uncertain polynomial systems, Automatica 40 (2004), no. 11, 1981-1986.

[CM18a] Justin Carpentier and Nicolas Mansard, Analytical derivatives of rigid body dynamics algorithms, Robotics: Science and Systems, 2018.

[CM18b] Multicontact locomotion of legged robots, IEEE Transactions on Robotics 34 (2018), no. 6, 1441-1460.

$\left[\mathrm{CSB}^{+}{ }^{+19]}\right.$ Justin Carpentier, Guilhem Saurel, Gabriele Buondonno, Joseph Mirabel, Florent Lamiraux, Olivier Stasse, and Nicolas Mansard, The Pinocchio C++ library - A fast and flexible implementation of rigid body dynamics algorithms and their analytical derivatives, IEEE International Symposium on System Integrations (SII), 2019.

[GH15] Peter Giesl and Sigurdur Hafstein, Review on computational methods for Lyapunov functions, Discrete and Continuous Dynamical Systems-Series B 20 (2015), no. 8, 2291-2331.

[GNS ${ }^{+}$17] Markus Giftthaler, Michael Neunert, Markus Stäuble, Marco Frigerio, Claudio Semini, and Jonas Buchli, Automatic differentiation of rigid body dynamics for optimal control and estimation, Advanced Robotics 31 (2017), no. 22, 12251237.

[GSM90] K Glover, J Sefton, and DC McFarlane, A tutorial on loop shaping using H-infinity robust stabilization, IFAC Proceedings Volumes 23 (1990), no. 8, 117-126.

[Joh00] Tor A Johansen, Computation of Lyapunov functions for smooth nonlinear systems using convex optimization, Automatica 36 (2000), no. 11, 1617-1626.

[Lib11] Daniel Liberzon, Calculus of variations and optimal control theory: a concise introduction, Princeton University Press, 2011.

[LK01] Steven M LaValle and James J Kuffner, Rapidly-exploring random trees: Progress and prospects, Algorithmic and Computational Robotics: New Directions (2001), no. 5, 293-308.

[Moc12] Jonas Mockus, Bayesian approach to global optimization: Theory and applications, vol. 37, Springer Science \& Business Media, 2012

[NN94] Yurii Nesterov and Arkadii Nemirovskii, Interior-point polynomial algorithms in convex programming, SIAM, 1994.

$\left[\mathrm{PGM}^{+} 19\right]$ Adam Paszke, Sam Gross, Francisco Massa, Adam Lerer, James Bradbury, Gregory Chanan, Trevor Killeen, Zeming Lin, Natalia Gimelshein, Luca Antiga, Alban Desmaison, Andreas Kopf, Edward Yang, Zachary DeVito, Martin Raison, Alykhan Tejani, Sasank Chilamkurthy, Benoit Steiner, Lu Fang, Junjie Bai, and Soumith Chintala, PyTorch: An imperative style, high-performance deep learning library, Advances in Neural Information Processing Systems 32, 2019 , pp. 8024-8035.

$\left[\mathrm{SCH}^{+} 18\right]$ Sumeet Singh, Mo Chen, Sylvia L Herbert, Claire J Tomlin, and Marco Pavone, Robust tracking with model mismatch for fast and safe planning: an SOS optimization approach, International Workshop on the Algorithmic Foundations of Robotics, Springer, 2018, pp. 545-564.

[SL91] Jean-Jacques E Slotine and Weiping Li, Applied nonlinear control, vol. 199, Prentice hall Englewood Cliffs, NJ, 1991.

[Sut96] Richard S Sutton, Generalization in reinforcement learning: Successful examples using sparse coarse coding, Advances in Neural Information Processing Systems, 1996, pp. 1038-1044.

[TMT11] Mark M Tobenkin, Ian R Manchester, and Russ Tedrake, Invariant funnels around trajectories using sum-of-squares programming, IFAC Proceedings Volumes 44 (2011), no. 1, 9218-9223.

[TMTR10] Russ Tedrake, Ian R Manchester, Mark Tobenkin, and John W Roberts, LOR-trees: Feedback motion planning via sums-of-squares verification, The International Journal of Robotics Research 29 (2010), no. 8, 1038-1052.

[TP07] Ufuk Topcu and Andrew Packard, Stability region analysis for uncertain nonlinear systems, IEEE Conference on Decision and Control, 2007, pp. 1693-1698. 


\section{APPENDiX A. ImPLEMENTATION SUMmary}

The complete ROA estimation and trajectory tracking frameworks are summarized respectively in Figure 4 and 5 Each building block used in the diagrams is detailed below. The first one computes the LQR as in Section 2 .

- Static LQR: $\quad\left(Q, R, x_{0}, u_{0}, f\right) \rightarrow(S, K)$.

$A=\frac{\partial f}{\partial x}\left(x_{0}, u_{0}\right), B=\frac{\partial f}{\partial u}\left(x_{0}, u_{0}\right), S$ is the positive definite solution of $A^{\top} S+S A-S B R^{-1} B^{\top} S=-Q$, and $K=$ $R^{-1} B^{\top} S$.

The following block computes the LQR for one time step of trajectory tracking and is detailed in Section 6 .

\section{- Dynamic LQR:}

$$
\left(S(t+\tau), Q, R, x_{0}(t), u_{0}(t), f\right) \rightarrow(S(t), K(t)) .
$$

Let $\bar{S}=S(t+\tau)$, then $S(t)=\bar{S}-\tau \dot{S}$, with $A=\frac{\partial f}{\partial x}\left(x_{0}(t), u_{0}(t)\right), B=\frac{\partial f}{\partial u}\left(x_{0}(t), u_{0}(t)\right)$,

$$
\dot{S}=-Q-\bar{S} A-A^{\top} \bar{S}+\bar{S} B R^{-1} B^{\top} \bar{S} \text {, and } K(t)=R^{-1} B^{\top} S(t) \text {. }
$$

The next two blocks compute bounds on the derivatives of the dynamics. They can be implemented arbitrarily.

- First-order oracle: $\left(\rho_{u p}, S, K, x_{0}, u_{0}, f\right) \rightarrow\left(A_{0}, V\right)$.

$$
V_{i j}:=\sup _{x^{\top} S x \leq \rho_{u p}}\left|J_{i j}(x)-\left(A_{0}\right)_{i j}\right|,
$$

where $J$ is the Jacobian of $x \mapsto f\left(x_{0}+x, u_{0}-K x\right)$. A default choice for $A_{0}$ is $J(0)=A-B K$.

- Second-order oracle: $\left(\rho_{u p}, S, K, M, x_{0}, u_{0}, f\right) \rightarrow U$.

$$
U_{i j}^{k}:=\sup _{x^{\top} S x \leq \rho_{u p}}\left[M^{-1 / 2} H^{k}(x) M^{-1 / 2}\right]_{i j},
$$

where $H$ is the Hessian of $x \mapsto f\left(x_{0}+x, u_{0}-K x\right)$.

The next two blocks compute stability certificates, as detailed in Sections 3 and 4.

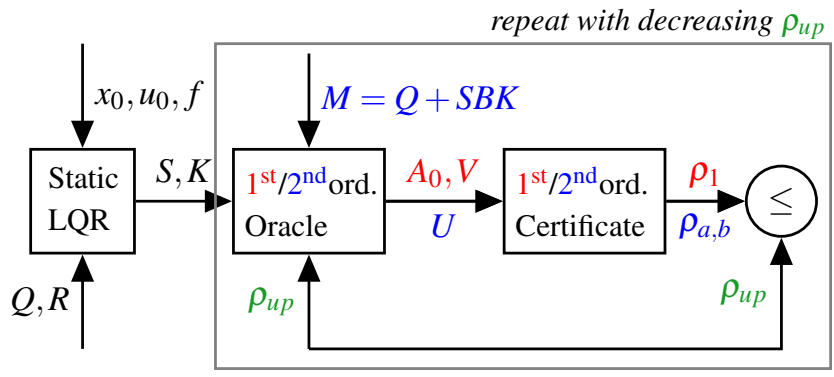

FIGURE 4. ROA estimation algorithm. Elements specific to the $1^{\text {st }}$ order method are in red, to the $2^{\text {nd }}$ order in blue. Framed steps are repeated until $\rho \geq \rho_{u p}$.

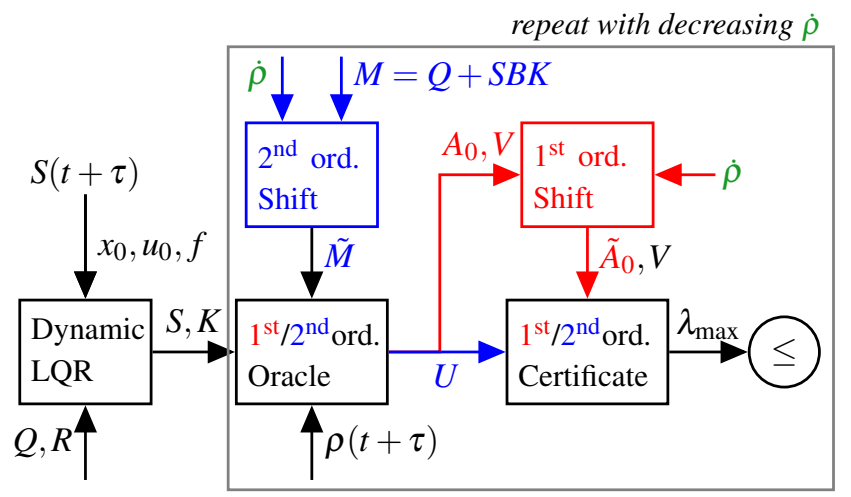

FIGURE 5. Trajectory tracking algorithm for one time-step. Framed steps are repeated while equations 16 for the $1^{\text {st }}$ order, or 17 for the $2^{\text {nd }}$ order, hold. 


\section{- First-order certificate:}

$$
\left(\rho_{u p}, S, A_{0}, V\right) \rightarrow \rho_{1}=\rho_{u p} \mathbf{1}_{\mathrm{LMI}} \text { is feasible } .
$$

Let $C, E$ defined as in section 3. The LMI feasibility problem is to find $\Lambda \succeq 0 \in \mathbb{R}^{d^{2} \times d^{2}}$ diagonal such that:

$$
\left[\begin{array}{cc}
A_{0}^{\top} S+S A_{0}+E^{\top} \Lambda E & S C \\
C^{\top} S & -\Lambda
\end{array}\right] \prec 0 .
$$

- Second-order certificate: $\left(\rho_{u p}, S, U\right) \rightarrow \rho_{a, b}=\frac{1}{\lambda_{a, b}^{2}}$.

$$
\begin{gathered}
\lambda_{a}=\lambda_{\max }\left(\sum_{k} \sqrt{S_{k} \cdot S^{-1} S_{k}^{\top}} U^{k}\right), \\
\lambda_{b}=\sqrt{d}\left\|D S^{-1 / 2}\right\|_{2}, \text { with } D=\operatorname{Diag}\left(\left\|U^{k}\right\|_{2}\right)_{k} .
\end{gathered}
$$

The last two blocks are used in Section 6$$
\text { - First-order shift: }\left(A_{0}, \dot{\rho}, \rho(t+\tau), S(t), \dot{S}(t)\right) \rightarrow \tilde{A}_{0} \text {. }
$$$$
\tilde{A}_{0}=A_{0}+\frac{1}{2} S^{-1} \dot{S}-\frac{1}{2} \frac{\dot{\rho}}{\rho} I_{d} \text {, where } \dot{S} \text { is given by the RDE (equation } 15 \text { ). }
$$

- Second-order shift:

$$
(M, \dot{\rho}, \rho(t+\tau), S(t)) \rightarrow \tilde{M}=M+\frac{\dot{\rho}}{\rho} S .
$$

